\title{
Multiple Neuronal Networks Mediate Sustained Attention
}

\author{
Natalia S. Lawrence ${ }^{*, 1}$, Thomas J. Ross ${ }^{\dagger, 1}$, Ray Hoffmann ${ }^{1}$, \\ Hugh Garavan ${ }^{2}$, and Elliot A. Stein ${ }^{\dagger, 1}$
}

\begin{abstract}
Sustained attention deficits occur in several neuropsychiatric disorders. However, the underlying neurobiological mechanisms are still incompletely understood. To that end, functional MRI was used to investigate the neural substrates of sustained attention (vigilance) using the rapid visual information processing (RVIP) task in 25 healthy volunteers. In order to better understand the neural networks underlying attentional abilities, brain regions where task-induced activation correlated with task performance were identified. Performance of the RVIP task activated a network of frontal, parietal, occipital, thalamic, and cerebellar regions. Deactivation during task performance was seen in the anterior and posterior cingulate, insula, and the left temporal and parahippocampal gyrus. Good task performance, as defined by better detection of target stimuli, was correlated with
\end{abstract}

\section{INTRODUCTION}

The rapid visual information processing (RVIP) task (Wesnes \& Warburton, 1983) is a serial target detection task that primarily requires sustained attention, but also working memory, for its execution. Deficits in sustained attention may underlie a number of cognitive abnormalities found in neuropsychiatric disorders, such as those in attention deficit hyperactivity disorder (Conners et al., 1996; Levin et al., 1996), Alzheimer's disease (White \& Levin, 1999; Jones, Sahakian, Levy, Warburton, \& Gray, 1992; Sahakian, Jones, Levy, Gray, \& Warburton, 1989), schizophrenia (Levin, Wilson, Rose, \& McEvoy, 1996), and Parkinson's disease (Kelton, Kahn, Conrath, \& Newhouse, 2000). Performance on sustained attention tasks, including the RVIP task, is impaired in these neuropsychiatric populations, but it can be improved by enhancing cholinergic transmission using cholinergic agonists such as nicotine (see Levin \& Rezvani, 2000 for a review). Thus, better understanding of the neural substrates of sustained attention is of importance not just to inform

\footnotetext{
${ }^{1}$ Medical College of Wisconsin, ${ }^{2}$ Trinity College, Dublin

*Current address: Section of Neuroscience and Emotion, Department of Psychological Medicine, Institute of Psychiatry, KCL, London SE5 8AF, UK.

${ }^{\dagger}$ Current address: Neuroimaging Research Branch, NIDA-IRP, 5500 Nathan Shock Drive, Baltimore, MD 21224, USA.
}

enhanced activation in predominantly right fronto-parietal regions and with decreased activation in predominantly left temporo-limbic and cingulate areas. Factor analysis revealed that these performance-correlated regions were grouped into two separate networks comprised of positively activated and negatively activated intercorrelated regions. Poor performers failed to significantly activate or deactivate these networks, whereas good performers either activated the positive or deactivated the negative network, or did both. The fact that both increased activation of task-specific areas and increased deactivation of task-irrelevant areas mediate cognitive functions underlying good RVIP task performance suggests two independent circuits, presumably reflecting different cognitive strategies, can be recruited to perform this vigilance task.

general theories of attention, but also to understand how pathology in neuropsychiatric disorders relates to cognitive function and, in turn, develop improved strategies to treat deficits in attention.

Previous lesion and neuroimaging studies have begun to delineate those regions that mediate specific aspects of attentional performance. Sustained attention has been ascribed predominantly to a right fronto-parietal-thalamic network (Sarter, Givens, \& Bruno, 2001; Coull, 1998; Coull, Frackowiak, \& Frith, 1998; Parasuraman, Warm, \& See, 1998). Specifically, the role of frontal regions, including the anterior cingulate, in target detection and executive control (Cabeza \& Nyberg, 2000; Posner, Petersen, Fox, \& Raichle, 1988; Posner \& Petersen, 1990), the right frontal and bilateral parietal areas in maintaining attention over time (Coull, 1998; Coull et al., 1998; Rueckert \& Grafman, 1996, 1998; Pardo, Fox, \& Raichle, 1991; Posner \& Petersen, 1990; Cohen et al., 1988; Deutsch, Papanicolaou, Bourbon, \& Eisenberg, 1987), and thalamic, midbrain, and reticular activating system structures in controlling cortical arousal (Coull, 1998; Marrocco \& Davidson, 1998; Paus et al., 1997; Kinomura, Larsson, Gulyas, \& Rolan, 1996) have been identified. Pharmacological enhancement of ascending cholinergic and noradrenergic projections from the basal forebrain and locus coeruleus, respectively, to much of the neocortex may help maintain cortical 
arousal and sustained and selective attention mechanisms in those individuals whose attentional performance is otherwise compromised.

Using positron emission tomography (PET), Coull, Frith, Frackowiak, and Grasby (1996) demonstrated that RVIP task performance is associated with activation in inferior frontal gyri, supplementary motor area (SMA), parietal cortex, and occipital/fusiform gyri. The present study sought to determine the neural basis of a novel block design version of the RVIP task using the superior temporal and spatial resolution of functional MRI (fMRI), and to determine those task-related activations that specifically correlate with task performance. Such correlational analysis can identify those regions that specifically underlie performance better than would a cognitive subtraction analysis. In the context of a correlational analysis in which all subjects are performing the task, relatively poor performers provide an excellent control group for relatively good performers, and regions whose activation increases as a parametric function of performance can be delineated. In addition, understanding which regional activation covaries with attentional abilities may guide investigations into functional differences between clinical populations and healthy individuals.

\section{RESULTS}

\section{Task Performance}

Complete behavioral and imaging details are given in the Methods. Subjects performed the sensorimotor control task at near-ceiling levels; the mean number $( \pm$ SEM) of targets detected (hits) was $93 \pm 1$ out of a possible 96 . The mean reaction time to hits was 480.28 $\pm 12.46 \mathrm{msec}$ and the mean number of false alarms was $0.56 \pm 0.26$. In contrast, the mean number of hits for the RVIP task was $60 \pm 4$ out of a maximum of 96 , whereas the mean reaction time to hits was $564.66 \pm$ $18.23 \mathrm{msec}$ and the mean number of false alarms was $5.32 \pm 1.35$. Paired $t$ tests revealed a significant difference between the control task and the RVIP task in both hits, $t(24)=9.68, p<.0001$, and reaction time to hits, $t(24)=6.08, p<.0001$. Thus, subjects performed the active task at about $65 \%$ of maximum and had a reaction time that was approximately $20 \%$ slower than during the control task. There were substantial individual differences in performance (the number of hits on the RVIP task ranged from 21 to 87), and this broad spread of data aided the regression analysis of performance against the BOLD data.

Performance on both tasks was examined for evidence of a vigilance decrement (decline in task performance over time). Repeated-measures ANOVA with polynomial trends analyses using the mean number of hits and reaction time per block as the repeated measures indicated that the number of hits in the RVIP task decreased as a linear function of time, $F(1,24)=5.66$, $p=.026$. Reaction time did not change significantly over the course of the RVIP task, although there was a linear trend towards an increase over time, $F(1,24)=3.1$, $p=.09$. In the control task, reaction time increased from Block 1 to Block 3 and then returned to baseline, an observation supported by the increase in reaction time as a cubic function of time, $F(1,24)=11.01$, $p=.003$. There was no change in the number of hits over time in the control task [linear trend: $F(1,24)=.73$, $p=.4]$.

\section{Functional Activation}

Comparison of whole brain BOLD activity during performance of the RVIP task to that during performance of the control task isolated 29 clusters of brain activation that differed significantly between the two task conditions. These clusters are detailed in Table 1. The largest clusters included bilateral activations of the inferior and superior parietal cortex, inferior and middle frontal gyri, pre-SMA, thalamus and caudate, anterior insula, and cerebellum (see Figure 1). All of these areas showed increased BOLD activity during performance of the RVIP task relative to the control task. There were also a number of areas that showed decreased BOLD activity during performance of the active task relative to control, including the anterior and posterior cingulate, left angular and middle temporal gyrus, mid-insula, left parahippocampal gyrus, and several small clusters in the left medial and middle frontal gyrus. It should be emphasized that these deactivations were the result of negative activation for both the RVIP and control tasks relative to the baseline state and were not a subtraction artifact of zero RVIP task activation.

Regression analyses revealed a number of areas (14/ 29) showing significant correlations between cluster activation and both number of hits and reaction time to hits (see Table 1). There were very few false alarms during RVIP task performance, and there were no significant correlations with false alarms. Of these 14 areas, nine showed significant positive correlations (i.e., increased activation associated with increased number of hits) including all right frontal regions, the left middle frontal gyrus, bilateral pre-SMA, bilateral parietal cortex, and the left anterior insula (see Figure 2). Five areas, all deactivated during the RVIP task, showed significant negative correlations with the number of hits (i.e., increased deactivation, as opposed to less positive activation, was associated with increased number of hits). These areas included the left medial frontal gyrus, bilateral anterior and posterior cingulate, the left middle temporal gyrus, and the right insula (see Figure 2). These data suggest that enhanced task performance, as measured by the number of targets detected, correlates with increased activation in predominantly right fronto-parietal networks, and with increased deactivation in predominantly left temporo-limbic areas. Most of these clusters are illustrated in Figure 1. 
Table 1. Clusters Identified as Showing Significant Differences in Activation between RVIP Task and the Control Task

\begin{tabular}{|c|c|c|c|c|c|c|}
\hline \multirow{2}{*}{$\begin{array}{l}\text { Brain Region (Cluster } \\
\text { Number in Figure 2) }\end{array}$} & \multirow[b]{2}{*}{ Side } & \multirow{2}{*}{$\begin{array}{c}\text { Center of Mass } \\
(x-y-z) R / L, A / P, S / I\end{array}$} & \multirow{2}{*}{$\begin{array}{l}\text { Brodmann's } \\
\quad \text { Area (S) }\end{array}$} & \multirow{2}{*}{$\begin{array}{l}\text { Cluster } \\
\text { Volume }(\mu l)\end{array}$} & \multicolumn{2}{|c|}{$\begin{array}{l}\text { BOLD-Performance } \\
\text { Correlation }\end{array}$} \\
\hline & & & & & Hits & $R T$ \\
\hline \multicolumn{7}{|l|}{ Frontal lobe } \\
\hline $\begin{array}{l}\text { Pre-SMA/medial and superior } \\
\text { frontal gyrus (19) }\end{array}$ & B & $1,15,45$ & $6,9,8,32$ & 5244 & $0.47^{*}$ & $-0.58^{* * *}$ \\
\hline $\begin{array}{l}\text { Middle and inferior frontal } \\
\text { gyrus (15) }\end{array}$ & $\mathrm{L}$ & $-43,10,29$ & $6,9,46$ & 4954 & 0.39 & $-0.54^{* * *}$ \\
\hline $\begin{array}{l}\text { Middle and inferior frontal } \\
\text { gyrus (16) }\end{array}$ & $\mathrm{R}$ & $44,4,31$ & 6,9 & 4401 & $0.49 *$ & $-0.61^{* *}$ \\
\hline $\begin{array}{l}\text { Middle and superior frontal } \\
\text { gyrus (17) }\end{array}$ & $\mathrm{R}$ & $42,27,24$ & 9,46 & 3241 & $0.48^{*}$ & -0.15 \\
\hline $\begin{array}{l}\text { Medial and middle frontal } \\
\text { gyrus (23) }\end{array}$ & $\mathrm{R}$ & $26,2,47$ & 6 & 3021 & $0.51 * *$ & $-0.56^{* * *}$ \\
\hline Middle frontal gyrus (22) & $\mathrm{L}$ & $-26,3,48$ & 6 & 710 & $0.46^{*}$ & $-0.4^{*}$ \\
\hline Superior, medial frontal gyrus & $\mathrm{L}$ & $-12,47,36$ & 8 & 701 & -0.28 & $0.41^{*}$ \\
\hline Middle, inferior frontal gyrus & $\mathrm{R}$ & $40,44,14$ & 10,46 & 557 & $0.48^{*}$ & -0.05 \\
\hline Medial frontal gyrus (9) & $\mathrm{L}$ & $-3,56,7$ & 10 & 405 & $-0.52 * *$ & 0.17 \\
\hline Middle frontal gyrus & $\mathrm{L}$ & $-29,20,38$ & 8 & 177 & -0.25 & 0.23 \\
\hline
\end{tabular}

\section{Parietal lobe}

Parietal lobule (inferior and superior, cuneus and

Parietal lobule (inferior and superior, precuneus and supramarginal gyrus) (20)

\section{Limbic/thalamic}

Anterior cingulate (8)

Posterior/middle cingulate (14)

Thalamus/basal ganglia (MD, VL, pulvinar nuclei of thalamus, caudate, putamen) (13)

\section{Insula}

Anterior insula (and inferior frontal gyrus/claustrum) (7)

Insula (mid-insula/claustrum) (12)

Anterior insula (10)

Insula (11)

Middle temporal gyrus (angular, superior temporal gyrus) (18)

$\mathrm{R}$

$\mathrm{R}$

L

$\mathrm{L}$

L
32, 18, 1

$$
38,-14,16
$$

$-31,18,3$

13

39, 19

$-38,-13,8$
1890

898

634

213

1121
0.34

$-0.47^{*}$

$-47,-60,26$
$-0.51 * *$

$0.58 * *$

0.38

$-0.12$

$-0.4^{*}$ 
Table 1. (continued)

\begin{tabular}{|c|c|c|c|c|c|c|}
\hline \multirow{2}{*}{$\begin{array}{l}\text { Brain Region (Cluster } \\
\text { Number in Figure 2) }\end{array}$} & \multirow[b]{2}{*}{ Side } & \multirow{2}{*}{$\begin{array}{l}\text { Center of Mass } \\
(x-y-z) R / L, A / P, S / I\end{array}$} & \multirow{2}{*}{$\begin{array}{l}\text { Brodmann's } \\
\text { Area }(S)\end{array}$} & \multirow{2}{*}{$\begin{array}{c}\text { Cluster } \\
\text { Volume }(\mu l)\end{array}$} & \multicolumn{2}{|c|}{$\begin{array}{c}\text { BOLD-Performance } \\
\text { Correlation }\end{array}$} \\
\hline & & & & & Hits & $R T$ \\
\hline \multicolumn{7}{|l|}{ Temporal lobe } \\
\hline $\begin{array}{l}\text { Parahippocampal } \\
\text { gyrus/culmen (6) }\end{array}$ & $\mathrm{L}$ & $-24,-32,-10$ & 36,35 & 464 & 0.05 & -0.13 \\
\hline $\begin{array}{l}\text { Angular and middle } \\
\text { temporal gyrus }\end{array}$ & $\mathrm{L}$ & $-45,-72,31$ & 39 & 179 & -0.27 & 0.21 \\
\hline \multicolumn{7}{|l|}{ Occipital lobe } \\
\hline $\begin{array}{l}\text { Occipital gyrus (inferior and } \\
\text { middle) and inferior } \\
\text { temporal gyrus (4) }\end{array}$ & $\mathrm{L}$ & $-44,-64,-5$ & $37,19,18$ & 4967 & 0.3 & $-0.42 *$ \\
\hline $\begin{array}{l}\text { Middle occipital gyrus } \\
\text { (inferior) }\end{array}$ & $\mathrm{R}$ & $29,-83,3$ & 18,19 & 397 & -0.24 & 0.02 \\
\hline $\begin{array}{l}\text { Middle occipital gyrus } \\
\text { (superior) }\end{array}$ & $\mathrm{L}$ & $-29,-86,11$ & & 218 & 0.032 & -0.16 \\
\hline \multicolumn{7}{|l|}{ Cerebellum } \\
\hline Cerebellum (culmen) (1) & $\mathrm{L}$ & $-30,-56,-24$ & & 2073 & 0.36 & -0.37 \\
\hline
\end{tabular}

The correlation between mean cluster activation and behavior (RVIP task performance) is from a simple regression of performance against activation. Numbers after cluster names correspond with numbers in Figure 1; areas without numbers are located between the illustrated slices. $\mathrm{B}=$ bilateral; $\mathrm{R}=$ right; $\mathrm{L}=$ left

$* p<.05$.

$* p<.01$.

When activation magnitude was regressed against reaction time to hits, good task performance (i.e., faster reaction times) was associated with increased activation in many of the same areas as those detailed above; namely the right medial and middle/inferior frontal gyrus, and the pre-SMA and bilateral parietal cortex. In addition, faster reaction times correlated with increased activation in the left frontal regions (left middle and superior frontal gyri), the left occipital/inferior temporal gyrus, bilateral cerebellum, and the right anterior insula/inferior frontal gyrus (see Figure 2). Only the left superior medial frontal gyrus (deactivated during RVIP task performance) showed a significant correlation between faster reaction times and decreased activations.

\section{Network Analysis}

A correlation matrix was generated to investigate the relationship between activations in the 29 defined clusters in all 25 subjects. A correlation coefficient $>.51$ was considered significant $(p<.01)$. The pattern that emerged pointed to an intercorrelated "network" of regions that was strongly activated during RVIP task performance, and a separate group of intercorrelated regions that was deactivated during task performance. 
There were no significant correlations between activations in these distinct "networks." High correlations also existed between activations in some homologous regions in the opposite hemisphere. For example, the left and right parietal cortices showed a correlation of .75, left and right middle/inferior frontal gyri (BAs 6 and 9) showed a correlation of .77 , and the left and right cerebellar culmen showed a correlation of 81 .

A subsequent factor analysis on the 29 clusters and hits identified three factors corresponding to three separate groups of interconnected regions. The first factor was composed of 15 clusters, each of which had a normalized coefficient of greater than 0.5 , and explained $32 \%$ of the variance. These regions are the parietal cortex (20-21), pre-SMA (19), middle/inferior frontal gyri (15-16), thalamus/caudate (13), occipital/ fusiform gyri (4-5), anterior insula $(7,10)$ (all bilateral), right medial/middle frontal gyrus (23), right middle/ superior frontal gyrus (17), left middle frontal gyrus (22), parahippocampal gyrus (6), and left middle occipital gyrus (not shown [NS]). The numbers in parentheses refer to the cluster number in Figure 1. Additionally, the number of hits had a large positive load on this factor (.54), and the regions listed above include eight of the nine regions positively correlated with the number of hits. Note that the ninth region, located in the right middle/inferior frontal gyrus (NS), had a normalized coefficient of 0.355 .
Figure 1. Regions showing differences in activation during RVIP and control task performance. Axial slices are taken every $9 \mathrm{~mm}$ from -21 $\mathrm{mm}$ (inferior) to $51 \mathrm{~mm}$ (superior). Red and yellow clusters show increased activation during RVIP task performance, blue clusters indicate deactivation (see color scale). 1 = left cerebellum, 2 = midline cerebellum, $3=$ right cerebellum, $4=$ left occipital gyrus, 5 = right fusiform gyrus, $6=$ left parahippocampal gyrus, $7=$ right anterior insula, $8=$ anterior cingulate, $9=$ left medial frontal cortex, $10=1 \mathrm{eft}$ anterior insula, $11=\mathrm{left}$ mid-insula, 12 = right mid-insula, $13=$ thalamus/ caudate, $14=$ posterior cingulate, $15=$ left middle/ inferior frontal gyrus, $16=$ right middle/inferior frontal gyrus, $17=$ right middle/superior frontal gyrus, $18=$ left middle temporal gyrus, $19=$ pre-SMA $20=$ left parietal, $21=$ right parietal, $22=$ left middle frontal gyrus, $23=$ right middle frontal gyrus. For more detailed anatomical descriptions of clusters shown, see Table 1.

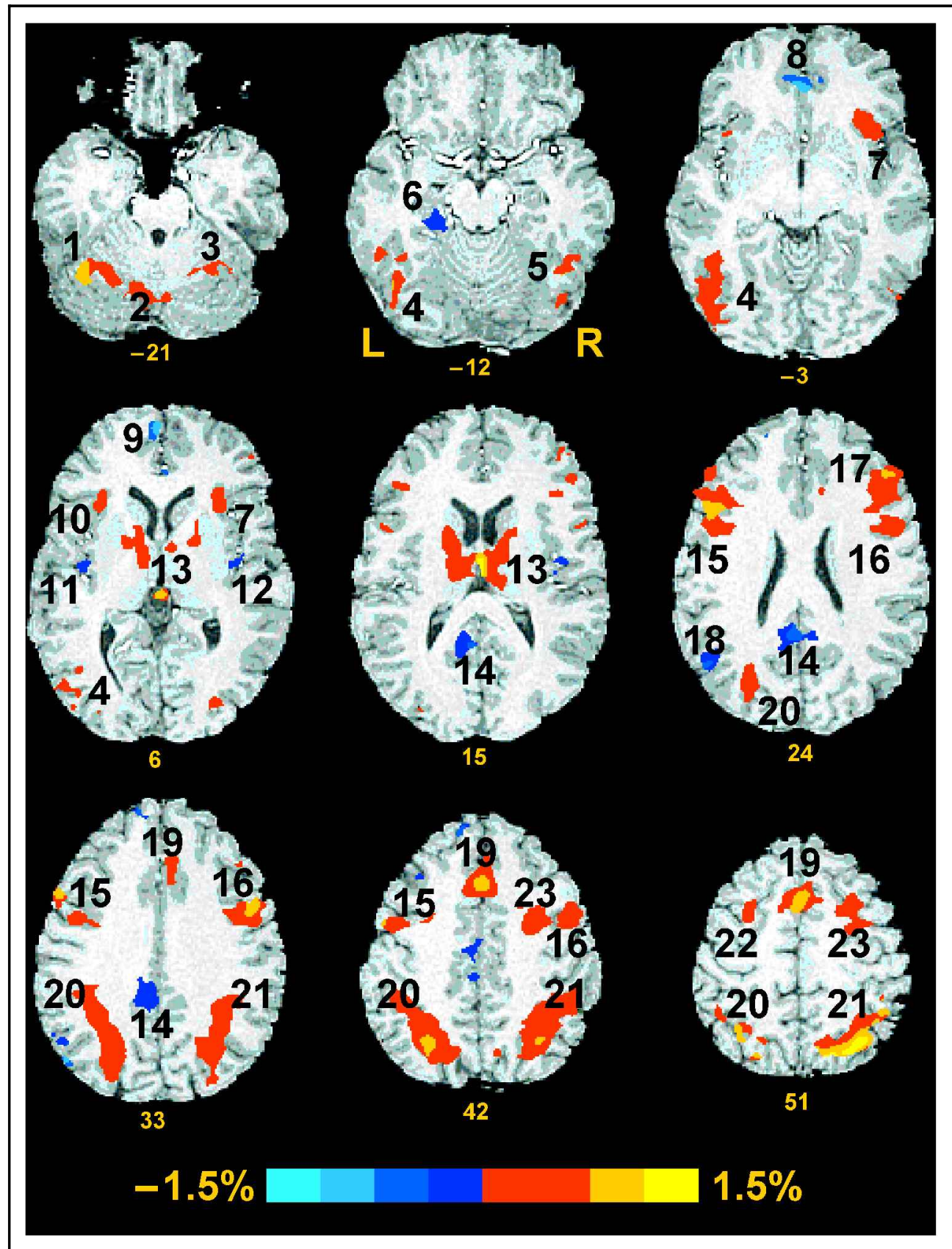


Figure 2. Correlations between cluster activation and performance. Graphs show correlations between change in activation (RVIP task - control) as a percent of baseline and the number of hits (top) and reaction time to hits (bottom). Annotations for regions are the same as for Figure 1. Correlation coefficients are indicated $(* p<.05, * * p<.01)$.

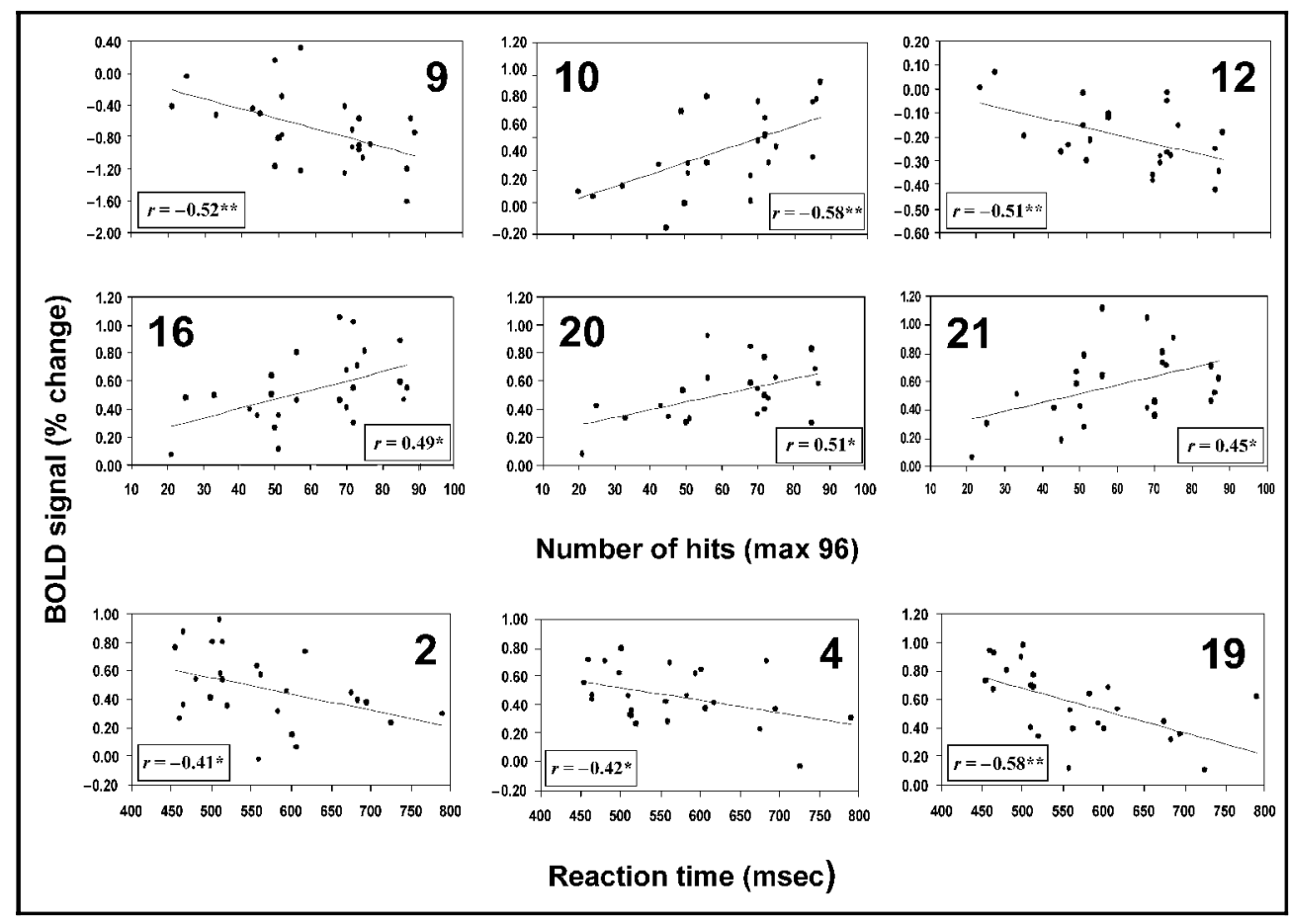

The second factor, explaining 19\% of the variance, consisted of nine regions that included the anterior cingulate (8), posterior/middle cingulate (14) (both bilateral), two left middle temporal and angular gyri clusters (18 and NS), three left medial and middle frontal gyri regions (9 and $2 \mathrm{NS}$ ), left insula (11) and right insula (12). All five of the regions that correlated negatively with the number of hits are included in this network. Additionally, the number of hits had a large negative load $(-.61)$ on this factor.

The third factor, explaining 9\% of the variance, included regions that did not correlate with the number of hits obtained in the RVIP task. Three cerebellar regions (1-3), activated during RVIP task performance, grouped together in this factor.

These analyses point to two central "networks" isolated by RVIP task performance; one showing positive changes in BOLD and one showing negative changes in BOLD. There were no direct correlations between the networks-either by examining the correlations directly or by examining factor loadings. The lack of correlations between activated and deactivated regions is surprising, considering that both magnitude of activation and deactivation are correlated with good task performance (see Table 1 and Figure 2). Together, these analyses suggest that in subjects who performed very well on the task, some strongly activated specific regions while others strongly deactivated regions, but there was no clear link between the two patterns of activation (i.e., strong "activators" were not necessarily strong "deactivators" and vice versa). To further investigate this possibility, we examined the relationship between amount of activation and deactivation in regions that significantly correlated with performance (number of hits) in all subjects. (Note that this does not include all regions included in the factor-analysis-defined networks outlined above; only those whose activation or deactivation correlated with the number of hits.)

Positive activation was summed across all nine clusters in the positively correlated network, and negative activation was summed across all six clusters in the negatively correlated network for each subject. Median splits were performed on the summed activation in each network, enabling subjects to be identified as being at the top or bottom half of "activators" or at the top or bottom half of "deactivators." This analysis indicated that of the 25 subjects, 7 were both top "activators" and top "deactivators" (i.e., showed strong activation and strong deactivation during the RVIP task), 6 were bottom "activators" and bottom "deactivators" (showed weak activation and weak deactivation during the task), 6 were top "activators" and bottom "deactivators" (showed strong activation but weak deactivation during the task), and the remaining 6 were bottom "activators" and top "deactivators" (showed weak task-related activation but strong deactivation). This simple median-split-based categorization of subjects into "activators," "deactivators," "mixed strong," and "mixed weak" types clearly shows an even spread across the four types. The nonparametric McNemar change test (Siegel \& Castellan, 1988) confirmed that there was no significant difference in the frequencies of subjects across these four types, $\chi^{2}=.083, p>.4$, demonstrating that strong "activators" are just as likely to be strong "deactivators" as they are to be weak "deactivators." This observation helps explain the lack 
of correlation between activations and deactivations across subjects.

The categorization of subjects into four response types was used to investigate demographic and/or performance differences between activators and deactivators. One-way ANOVA indicated that the four groups of "activator"-type did not differ in terms of age or years of education, although there was a significant group effect on performance, $F(3,24)=9.54, p<.001$. Post hoc Bonferroni tests revealed that subjects falling into the bottom half of both activation and deactivation performed significantly more poorly than subjects in the three other groups ( $p \leq .01$ for all comparisons), consistent with the bivariate correlations reported above between activation/deactivation and performance. Subjects in the "weak activation, weak deactivation" group obtained an average of 36 hits, whereas subjects in the other three groups obtained an average of 63-70 hits.

\section{DISCUSSION}

Subjects performed the novel block design RVIP task with varying levels of success, but the mean number of hits (as a percent of total) was similar to those found in previous studies using the more common continuous presentation version (Foulds et al., 1996; Sahakian et al., 1989). The fact that the number of hits while performing the RVIP task decreased as a function of time within a session supports a performance deficit in this modified version of the task and is consistent with that expected from a high demand on sustained attention (Parasuraman et al., 1998).

Relative to the control task, the RVIP task produced a distributed network of cerebral activation composed of frontal, parietal, thalamic, caudate, occipital, and cerebellar activations previously associated with visual attention and working memory (Cabeza \& Nyberg, 2000; Garavan, Ross, Li, \& Stein, 2000; Coull, 1998; D'Esposito et al., 1998; Jonides et al., 1998; Rueckert \& Grafman, 1996, 1998; Pardo et al., 1991; Cohen et al., 1988; Posner et al., 1988; Posner \& Petersen, 1990; Deutsch et al., 1987). In addition, strong task-induced activation of the anterior insula was observed. Correlation analysis of task performance with BOLD activation magnitude demonstrated that a large number of fronto-parietal regions were positively correlated with number of hits and faster reaction times to hits. The strongest positive correlations between activation magnitude and number of hits were found in the left anterior insula, left parietal cortex, and right frontal regions. Activation in the right frontal cortex and bilateral pre-SMA showed the strongest positive correlations with faster reaction times.

Based on earlier studies, it is likely that the observed right frontal, bilateral lateral prefrontal (middle/inferior frontal gyrus; BAs 6, 9, and 46), and parietal cortical activations were a consequence of both the sustained attention loading of the RVIP task (Coull, 1998; Rueckert \& Grafman, 1996, 1998; Pardo et al., 1991; Posner \& Petersen, 1990; Cohen et al., 1988; Deutsch et al., 1987) and the task's working memory demands, including maintenance, updating, and verification of serial stimuli (Cabeza \& Nyberg, 2000; Garavan et al., 2000; D'Esposito et al., 1998; Baker et al., 1996; Cohen et al., 1994). Parietal cortex activation has also been associated with executive functions such as allocation of attention (Garavan et al., 2000) and verbal working memory (Jonides et al., 1998). The presently observed right and left parietal cortex activation during RVIP task performance could be linked to, respectively, activation of Baddeley's (1986) visuospatial sketchpad and phonological loop components of working memory, employed to hold digits online (Coull et al., 1996). Although data from previous studies enable us to speculate on the functional significance of performance-correlated activations and deactivations in this study, it is difficult to draw specific conclusions because not only are many regions identified here similar to those engaged by a variety of difficult tasks (Duncan \& Owen, 2000), but also, the study's block design, long TR, and, critically, the nature of the task, which lacks the distinct "attentional" events that would make it amenable to an eventrelated design, prevented us from identifying brain activation associated with specific aspects of RVIP task performance. A different event-related design, employing a shorter TR, would potentially enable delineation of BOLD responses associated with, for example, correct identification of targets versus processing of nontargets, or areas showing a decline in activation over time (i.e., mediating the vigilance decrement), such as in Coull et al., 1998.

The strong correlation between left anterior insula activation and attention performance has not been previously documented but perhaps is not surprising given the reciprocal connections between the anterior/ mid-insula and both prefrontal (BA 6) and parietal regions (Augustine, 1996) that have also been implicated in RVIP task performance. Along with its established role in taste perception and visceral somatosensory processing, more cognitive roles for the insula in selective visual attention (Corbetta, Miezin, Dobmeyer, Shulman, \& Petersen, 1991), verbal working memory (Paulesu, Frith, \& Frackowiak, 1993), and linguistic processing (Augustine, 1996; Habib et al., 1995) have previously been suggested. Alternatively, anterior insula activation could be explained by a possible increase in arousal/anxiety during blocks of the demanding RVIP task versus the less anxiety-provoking control task, with increased arousal producing enhanced task performance. This interpretation could be addressed in the future by using skin conductance and mood measures during task performance to monitor explicit and autonomic measures of anxiety/arousal and determine their correlations with RVIP task performance. 
RVIP task performance was also associated with deactivation in left frontal, ventral medial prefrontal, anterior and posterior cingulate, mid/posterior insula, and left parahippocampal regions. These BOLD signal decreases occurred both relative to the control task and relative to the intertask resting state (data not presented). Activation in almost all of these regions showed significant negative correlations with the number of hits identified in the RVIP task, suggesting that deactivation may be beneficial to successful task performance. The strongest negative correlations between the number of hits and mean cluster activation were found in the right mid-insula and left medial frontal gyrus, suggesting that sustained attention is best when these two areas are relatively suppressed, perhaps reflecting distraction or attention interference as a result of ongoing neural processing in these areas.

Many of the deactivated regions (ventral medial front$\mathrm{al}$, anterior and posterior cingulate) have high metabolic activity at rest (Raichle et al., 2001) and have repeatedly shown deactivation (measured with PET and fMRI) during the performance of demanding goal-directed tasks that require attending to external stimuli (Gusnard \& Raichle, 2001; Shulman et al., 1997). The significance of deactivations during performance of such tasks relative to a passive baseline state is unclear, but may reflect the inhibition of processes such as monitoring of the environment and/or internal mood state that are ongoing both at rest and during a relatively simple control task, and which interfere with task performance (Gusnard \& Raichle, 2001). Indeed, many of the currently identified deactivated regions have been shown to be involved in emotional and somatosensory processing (Simpson, Snyder, Gusnard, \& Raichle, 2001; Maddock, 1999), as well as "conceptual" processing, such as retrieval of semantic knowledge (Binder et al., 1999), retrieval of episodic memories (Cabeza \& Nyberg, 2000), and selfreferential mental processes (Gusnard, Akbudak, Shulman, \& Raichle, 2001). Decreased activation in anterior and posterior cingulate, left medial frontal gyrus, and mid-insula during RVIP task performance, therefore, could be related to the need for focused attention towards the specific task demands as opposed to a more diffuse distribution of attention to introspective and emotional factors during the "resting" state (Gusnard \& Raichle, 2001; Coull et al., 1996). In support of this conjecture, McKiernan, Kaufman, Kucera-Thompson, and Binder (2003) found that deactivation in many of these same areas increases as exogenous task demands increase, presumably reflecting a greater need for focused attention towards more difficult tasks.

The present data suggest that good performance on the RVIP task is associated with increased activation in a fronto-parietal network underlying attention and working memory, and decreased activation in more limbic left medial frontal, temporal, and cingulate regions. Activation in the clusters positively associated with task performance showed high intercorrelations and were grouped into a positively activated network, which accounted for a third of the variance based on factor analysis. The clusters negatively associated with task performance showed similar intercorrelations, were grouped into a negatively activated network, and displayed the second highest factor loading. The lack of direct correlations or relationships between the distinct positively activated and negatively activated networks call into question simple resource reallocation models of deactivation, which posit that due to limited processing capacity, in order to enhance processing and taskinduced activation in one network (e.g., underlying perceptual processing), it is necessary to decrease activation in another (e.g., mediating "conceptual" processing; Binder et al., 1999). However, our findings did indicate that both magnitude of activation and deactivation were associated with task performance because both correlated with the number of hits.

In addition, it seemed that good performance could arise from stronger activation in the positive performance-associated areas, stronger deactivation in the negative performance-associated areas, or a mixture of strong levels of activation and deactivation. Weak levels of activation and deactivation during the RVIP task seem to be related to poorer task performance. This finding points to the intriguing possibility that increasing attention and working memory during task performance can be accomplished by distinct neurobiological strategies; either by predominantly boosting prefrontal-parietal cortex activity, predominantly deactivating temporolimbic circuitry, or a combination of the two. These different patterns of brain response could arise from individual differences in "default" brain activity at rest or during the unchallenging control tasks, so that changes in BOLD induced by difficult tasks will either resemble "deactivation" if activity is high at baseline, or "activation" if the brain activity at baseline is low. The nature and cause of individual differences in regional activations and deactivations during the performance of demanding cognitive tasks beg more detailed investigation and invite an alternative way of examining the neurobiological basis of cognitive dysfunction in clinical populations.

\section{METHODS}

\section{Subjects}

Twenty-five healthy, right-handed (Edinburgh Handedness Inventory, Oldfield, 1971) volunteers participated in this study. Subjects included 15 women and 10 men aged between 18 and 36 years (mean age $=24.3$ years, $S D=5.5)$ with a mean education of 15.2 years $(S D=$ 2.8). Subjects had no previous history of any neurological or psychiatric disorder and drug dependence (including nicotine use). After complete description of the study, subjects gave written informed consent to the Medical College of Wisconsin Institutional Review Board 
approved protocol. Subjects were asked to abstain from alcohol consumption for $8 \mathrm{hr}$ prior to the study. Caffeine withdrawal was avoided by asking caffeine-using subjects to consume one half-cup of caffeine-containing beverage at least $2 \mathrm{hr}$ prior to the study. A urine sample was obtained immediately prior to the study and absence of drug use confirmed with an ELISA assay $\left(\right.$ TRIAGE $^{\circledR}$ ).

\section{Task}

Subjects performed a block design version of the RVIP task. The task was composed of 90 -sec blocks of continuous stimuli presentation, consisting of a stream of single digits presented in the center of a computer screen at a rate of 100 digits/min. During the RVIP task, subjects were instructed to press a button using their right index finger when they saw a target sequence of three different odd or three different even numbers appearing consecutively. The target sequences were not composed of specific ascending or descending sequences, but of any three odd or even digits, that is, $7-1-3$ was a target sequence, as was 2-4-6. Subjects were encouraged to be accurate and were informed that a correct response to a target sequence (a "hit") would be recorded for up to $1200 \mathrm{msec}$ after the target sequence (i.e., during the two digits following a target sequence). Each block contained 12 pseudorandomly occurring targets, with four targets appearing every $30 \mathrm{sec}$. Targets were always separated by at least two digits, and the same digit never appeared twice consecutively. Eight blocks of RVIP task were performed consecutively with eight blocks of a low-level visuomotor control task, wherein subjects were instructed to press a button using their right index finger when they saw the number "0" appear on the screen. The stream of digits was identical to that used in the RVIP task, with one of every three digits in the target sequences being replaced by " 0 ." The " 0 " only appeared in the control task. The sequence of the two tasks was presented in an $\mathrm{AB} / \mathrm{BA}$ pseudorandom order and was followed by a 30-sec rest period, during which subjects were instructed to fixate on a cross in the center of the screen. Subjects received a visual warning of which task type they were about to perform immediately prior to the block presentation. A reminder of the target type in the current block was also displayed at the bottom of the screen throughout the task. Task performance was assessed by calculating the number of targets detected (hits), false alarms, and the mean reaction time (msec) to target stimuli for each scan session. These performance indices were calculated separately for the control and the RVIP task.

The 28-min block design version of the task was first tested outside the MRI in a separate group of subjects to ensure that the inclusion of rest periods and the control task did not facilitate task performance relative to the traditional continuous 10-min version of the RVIP task. Such an improvement could have arisen from the possible alleviation of attention decrement due to the rest periods in the block design task. Results of a counterbalanced within-subjects study on eight separate control subjects indicated no significant differences between the two task versions, as assessed by a repeated-measures ANOVA on the total number of hits obtained, $F(1,7)=.59, p=.814$, and the mean reaction time to hits, $F(1,7)=.00009$, $p=.99$. All subjects were trained on the task once outside of the scanner prior to their scanning session. None of the pilot subjects participated in the scanning experiment.

\section{fMRI Parameters}

Scanning was performed on a 3T Bruker Medspec scanner equipped with a 30.5-cm-i.d., three-axis local gradient coil and an endcapped quadrature birdcage radio frequency head coil (Wong, Boskamp, \& Hyde, 1992). Contiguous 4-mm sagittal slices covering the entire brain were collected using a blipped gradient-echo, echoplanar pulse sequence $(\mathrm{TE}=27.2 \mathrm{msec}$; $\mathrm{TR}=6000 \mathrm{msec}$; FOV $=24 \mathrm{~cm} ; 64 \times 64$ matrix; $3.75 \times 3.75 \mathrm{~mm}$ in-plane resolution). Foam padding was used to limit head movements inside the coil. High-resolution inversion-recovery anatomical images (MPRAGE) were acquired following functional imaging to enable subsequent localization of functional activation. Stimuli were back projected onto a screen at the subject's feet and were viewed with the aid of prism glasses attached to the inside of the RF head coil.

\section{fMRI Analyses}

Data processing was conducted with the software package AFNI v.2.4 (Cox, 1996). Three-dimensional volumetric motion correction and edge detection algorithms were first applied to the EPI data. No subject showed significant residual motion, thus allowing for data from all 25 subjects to be included in the data analysis. A multiple regression analysis was applied to the EPI data, with regressors corresponding to the blocks of RVIP task, blocks of control task and the six motion correction parameters. The change in signal produced during the blocks of RVIP task and during the blocks of control task was calculated relative to the baseline signal during rest. A subtraction map was also generated in which the changes in signal produced during the blocks of control task were subtracted from signal changes during RVIP task blocks.

Activation maps were converted to a standard stereotaxic coordinate system (Talairach \& Tournoux, 1988) and spatially blurred ( $4.2 \mathrm{~mm}$ FWHM isotropic Gaussian filter). Functional regions of interest (ROIs) were generated using data from a subtraction analysis (RVIP task control task) by calculating one-sample $t$ tests against the null hypothesis of no difference on the mean per voxel signal differences across subjects. The $t$ test map was thresholded using an alpha value calculated by a randomization test based on all the subjects' subtraction data. The randomization generated 500 pseudoreplicate data files by taking each subject's data and randomly reversing the sign of the change in BOLD signal with a 
probability of .5. Based upon the frequency of resulting false-positives, the randomization generated a $t$ value (4.7) corresponding to an omnibus $p$ value of .05 . Additionally, significant voxels had to be grouped into contiguous clusters of a minimum volume of $150 \mu \mathrm{l}$, approximately three times the size of the originally acquired voxels (Forman et al., 1995).

A mask was created composed of 29 clusters that showed a significant difference in activation between the control task and the RVIP task (Table 1). The functional ROI mask was applied to each subject's subtraction data to determine the average RVIP-induced activation value per cluster. Simple regression analyses were performed using Statview (SAS, Cary, NC) to assess the relationship between the behavioral data (number of hits, mean reaction time to hits, and false alarms for each subject) and the fMRI data (mean task-induced brain activation per cluster from each subject). In addition, a correlation matrix was generated using mean activation values from all clusters to determine which ROIs showed intercorrelated levels of task-induced activation. Intercorrelated cluster activations were further grouped together by applying a factor analysis to the data using SAS (Hatcher, 1994).

Factor analysis was used to identify how the activated ROIs and the performance on the RVIP task were related. Exploratory factor analysis assumes that there is an underlying causal structure that is reflected in the observed ROI due to the presence of one or more latent (unobserved) variables. As applied to fMRI data, the "factors" represent networks of regions that are associated with activation as a result of the RVIP task.

\section{Acknowledgments}

Supported by NIDA grants DA 09465 and DA 141000 and GCRC grant RR00058. The assistance of Drs. Giovanna Mancuso and Catherine Harmer for design consultations, and T. Maestas and S. Engelbart for technical assistance, is gratefully acknowledged.

Reprint requests should be sent to Elliot A. Stein, PhD, Chief, Neuroimaging Research Branch, NIDA-IRP, 5500 Nathan Shock Drive, Baltimore, MD 21224, USA, or via e-mail: estein@intra. nida.nih.gov.

The data reported in this experiment have been deposited with The fMRI Data Center archive (http://www.fmridc.org). The accession number is 2-2003-113K3.

\section{REFERENCES}

Augustine, J. R. (1996). Circuitry and functional aspects of the insular lobe in primates including humans. Brain Research Reviews, 22, 229-244.

Baddeley, A. (1986). Working memory. Oxford, UK: Clarendon Press.

Baker, S. C., Rogers, R. D., Owen, A. M., Frith, C. D., Dolan, R. J., Frackowiak, R. S. J., \& Robbins, T. W. (1996). Neural systems engaged by planning: A PET study of the Tower of London task. Neuropsychologia, 34, 515-526.

Binder, J. R., Frost, J. A., Hammeke, T. A., Bellgowan, P. S. F.,
Rao, S. M., \& Cox, R. W. (1999). Conceptual processing during the conscious resting state: A functional MRI study. Journal of Cognitive Neuroscience, 11, 80-93.

Cabeza, R., \& Nyberg, L. (2000). Imaging cognition: II. An empirical review of 275 PET and fMRI studies. Journal of Cognitive Neuroscience, 12, 1-47.

Cohen, J. D., Forman, S. D., Braver, T. S., Casey, B. J., Servan-Schreiber, D., \& Noll, D. C. (1994). Activation of the prefrontal cortex in a nonspatial working memory task with functional MRI. Human Brain Mapping, 1, 293-304.

Cohen, R. M., Semple, W. E., Gross, M., Holcomb, H. H., Dowling, M. S., \& Nordahl, T. E. (1988). Functional localization of sustained attention: Comparison to sensory stimulation in the absence of instruction. Neuropsychiatry, Neuropsychology and Behavioral Neurology, 1, 3-20.

Conners, C. K., Levin, E. D., Sparrow, E., Hinton, S. C., Erhardt, D., Meck, W. H., Rose, J. E., \& March, J. (1996). Nicotine and attention in adult ADHD. Psychopharmacological Bulletin, 32, 67-73.

Corbetta, M., Miezin, F. M., Dobmeyer, S., Shulman, G. L., \& Petersen, S. E. (1991). Selective and divided attention during visual discriminations of shape, color and speed: Functional anatomy by Positron Emission Tomography. Journal of Neuroscience, 11, 2383-2402.

Coull, J. T. (1998). Neural correlates of attention and arousal: Insights from electrophysiology, functional neuroimaging and psychopharmacology. Progress in Neurobiology, 55, 343-361.

Coull, J. T., Frackowiak, R. S., \& Frith, C. D. (1998). Monitoring for target objects: Activation of right frontal and parietal cortices with increasing time on task. Neuropsychologia, 36, $1325-1334$.

Coull, T., Frith, C. D., Frackowiak, R. S. J., \& Grasby, P. M. (1996). A fronto-parietal network for rapid visual information processing: A PET study of sustained attention and working memory. Neuropsychologia, 34, 1085-1095.

Cox, R. W. (1996). AFNI: Software for the analysis and visualization of functional magnetic resonance neuroimages. Computer and Biomedical Research, 29, 162-173.

D'Esposito, M., Aguirre, G. K., Zarahn, E., Ballard, D., Shin, R. K., \& Lease, J. (1998). Functional MRI studies of spatial and nonspatial-working memory. Cognitive Brain Research, 7, $1-13$.

Deutsch, G., Papanicolaou, A. C., Bourbon, W. T., \& Eisenberg, H. M. (1987). Cerebral blood flow evidence of right frontal activation in attention demanding tasks. International Journal of Neuroscience, 36, 23-28.

Duncan, J., \& Owen, A. M. (2000). Common regions of the human frontal lobe recruited by diverse cognitive demands. Trends in Neurosciences, 23, 475-483.

Forman, S. D., Cohen, J. D., Fitzgerald, M., Eddy, W. F., Mintun, M. A., \& Noll, D. C. (1995). Improved assessment of significant activation in functional magnetic resonance imaging (fMRI): Use of a cluster-size threshold. Magnetic Resonance in Medicine, 33, 636-647.

Foulds, J., Stapleton, J., Swettenham, J., Bell, N., McSorley, K., \& Russell, M. A. H. (1996). Cognitive performance effects of subcutaneous nicotine in smokers and never-smokers. Psychopharmacology, 127, 31-38.

Garavan, H., Ross, T. J., Li, S.-J., \& Stein, E. A. (2000). A parametric manipulation of central executive functioning. Cerebral Cortex, 10, 585-592.

Gusnard, D. A., Akbudak, E., Shulman, G. L., \& Raichle, M. E. (2001). Medial prefrontal cortex and self-referential mental activity: Relation to a default mode of brain function. Proceedings of the National Academy of Sciences, U.S.A., 98, 4259-4264. 
Gusnard, D. A., \& Raichle, M. E. (2001). Searching for a baseline: Functional imaging and the resting human brain. Nature Neuroscience Reviews, 2, 685-694.

Habib, M., Daquin, G., Milandre, L., Royere, M. L., Rey, M., Lanteri, A., Salame, G., \& Khalil, R. (1995). Mutism and auditory agnosia due to bilateral insular damage-role of the insula in human communication. Neuropsychologia, 33, $327-339$.

Hatcher, L. (1994). Step by step approach to using the SAS system for factor analysis and structural equation modeling. Cary, NC: SAS Institute Press.

Jones, G. M. M., Sahakian, B. J., Levy, R., Warburton, D. M., \& Gray, J. A. (1992). Effects of sub-cutaneous nicotine on attention, information processing and short-term memory in Alzheimer's Disease. Psychopharmacology, 108, 485-494.

Jonides, J., Schumacher, E. H., Smith, E. E., Koeppe, R. A., Awh, E., Reuter-Lorenz, P. A., Marshuetz, C., \& Willis, C. R. (1998). The role of parietal cortex in verbal working memory. Journal of Neuroscience, 18, 5026-5034.

Kelton, M. C., Kahn, H. J., Conrath, C. L., \& Newhouse, P. A. (2000). The effects of nicotine on Parkinson's disease. Brain and Cognition, 43, 274-282.

Kinomura, S., Larsson, J., Gulyas, B., \& Rolan, P. E. (1996). Activation by attention of the human reticular formation and thalamic intralaminar nuclei. Science, 271, 512-515.

Levin, E. D., Conners, C. K., Sparrow, E., Hinton, S. C., Erhardt, D., Meck, W. H., Rose, J. E., \& March, J. (1996). Nicotine effects on adults with attention-deficit/hyperactivity disorder. Psychopharmacology, 123, 55-63.

Levin, E. D., \& Rezvani, A. H. (2000). Development of nicotinic drug therapy for cognitive disorders. European Journal of Pharmacology, 393, 141-146.

Levin, E. D., Wilson, W., Rose, J., \& McEvoy, J. (1996). Nicotine-haloperidol interactions and cognitive performance in schizophrenics. Neuropsychopharmacology, 15 , 429-436.

Maddock, R. J. (1999). The retrosplenial cortex and emotion: New insights from functional neuroimaging of the human brain. Trends in Neurosciences, 22, 310-316.

Marrocco, R. T., \& Davidson, M. C. (1998). Neurochemistry of attention. In R. Parasuraman (Ed.), The attentive brain (pp 35-50). Cambridge: MIT Press.

McKiernan, K. A., Kaufman, J. N., Kucera-Thompson, J., \& Binder, J. R. (2003). A parametric manipulation of factors affecting task-induced deactivation in functional neuroimaging. Journal of Cognitive Neuroscience, 15, $394-408$

Oldfield, R. C. (1971). The assessment and analysis of handedness: The Edinburgh Inventory. Neuropsychologia, 9, 97-113.

Parasuraman, R., Warm, J. S., \& See, J. E. (1998). Brain systems of vigilance. In R. Parasuraman (Ed.), The attentive brain (pp. 221-256). Cambridge: MIT Press.

Pardo, J. V., Fox, P. T., \& Raichle, M. E. (1991). Localization of a human system for sustained attention by positron emission tomography. Nature, 349, 61-64.

Paulesu, E., Frith, C. D., \& Frackowiak, R. S. J. (1993). The neural correlates of the verbal component of working memory. Nature, 362, 342-344.

Paus, T., Zatorre, R. J., Hofle, N., Caramanos, Z., Gotman, J., Petrides, M., \& Evans, A. C. (1997). Time-related changes in neural systems underlying attention and arousal during the performance of an auditory vigilance task. Journal of Cognitive Neuroscience, 9, 392-408.

Posner, M. I., \& Petersen, S. E. (1990). The attention system of the human brain. Annual Review of Neuroscience, 13, 25-42.

Posner, M. I., Petersen, S. E., Fox, P. T., \& Raichle, M. E. (1988). Localization of cognitive operations in the human brain. Science, 240, 1627-1631.

Raichle, M. E., MacLeod, A. M., Snyder, A. Z., Powers, W. J., Gusnard, D. A., \& Shulman, G. L. (2001). A default mode of brain function. Proceedings of the National Academy of Sciences, U.S.A., 98, 676-682.

Rueckert, L., \& Grafman, J. (1996). Sustained attention deficits in patients with right frontal lesions. Neuropsychologia, 34, 953-963.

Rueckert, L., \& Grafman, J. (1998). Sustained attention deficits in patients with lesions of posterior cortex. Neuropsychologia, 36, 653-660.

Sahakian, B., Jones, G., Levy, R., Gray, J., \& Warburton, D. (1989). The effects of nicotine on attention, information processing, and short-term memory in patients with dementia of the Alzheimer type. British Journal of Psychiatry, 154, 797-800.

Sarter, M., Givens, B., \& Bruno, J. P. (2001). The cognitive neuroscience of sustained attention: Where top-down meets bottom-up. Brain Research Reviews, 35, 146-160.

Shulman, G. L., Fiez, J. A., Corbetta, M., Buckner, R. L., Miezin, F. M., Raichle, M. E., \& Petersen, S. E. (1997). Common blood flow changes across visual tasks: II. Decreases in cerebral cortex. Journal of Cognitive Neuroscience, 9, 648-663.

Siegel, S., \& Castellan, N. J., Jr. (1988). Nonparametric statistics for the behavioural sciences. New York: McGraw-Hill.

Simpson, J. R. J., Snyder, A. Z., Gusnard, D. A., \& Raichle, M. E. (2001). Emotion-induced changes in human medial prefrontal cortex: I. During cognitive task performance. Proceedings of the National Academy of Sciences, U.S.A., 98, $683-687$

Talairach, J., \& Tournoux, P. (1988). Co-planar stereotaxic atlas of the buman brain. New York: Thieme.

Wesnes, K., \& Warburton, D. M. (1983). The effects of smoking on rapid information processing performance. Neuropsychobiology, 9, 223-229.

White, H. K., \& Levin, E. D. (1999). Four-week nicotine skin patch treatment effects on cognitive performance in Alzheimer's disease. Psychopharmacology, 143, $158-165$.

Wong, E. C., Boskamp, E., \& Hyde, J. S. (1992). A volume optimized quadrature elliptical endcap birdcage brain coil. Berlin, 11th Annual Scientific Meeting, Society for Magnetic Resonance Medicine, 4015. 